\title{
Anti-glomerular Basement Membrane Disease Presenting as Hypertensive Emergency and Seizures
}

\author{
Michael Mascola ${ }^{1}$, Rehan Karmali ${ }^{2}$, Joshua Mathews ${ }^{2}$, and Sergio Obligado ${ }^{2}$ \\ ${ }^{1}$ Touro College of Osteopathic Medicine \\ ${ }^{2}$ Orange Regional Medical Center
}

June 11, 2020

\begin{abstract}
We present a 30-year-old male with end-stage renal disease (ESRD) secondary to anti-glomerular basement membrane (antiGBM) disease who presented to the emergency room with hypertensive emergency and seizures. He was treated with antihypertensives and lorazepam. His seizure etiology was attributed to hypertensive emergency due to ESRD from anti-GBM disease.
\end{abstract}

Anti-glomerular Basement Membrane Disease Presenting as Hypertensive Emergency and Seizures

Michael Mascola ${ }^{1,2}$, Rehan Karmali ${ }^{1,2}$, Joshua Mathews $^{2}$, Sergio Obligado ${ }^{2}$

${ }^{1}$ Touro College of Osteopathic Medicine, Middletown NY

${ }^{2}$ Orange Regional Medical Center, Middletown NY

Corresponding/first Author Contact:

Michael Mascola, OMS-III

Orange Regional Medical Center

707 E Main St, Middletown NY 10940

Email:mmascola@student.touro.edu

Cell Phone: 203-942-4053

\section{KEY CLINICAL MESSAGE}

Anti-glomerular basement membrane disease can rapidly lead to renal failure and dysregulation of blood pressure. A rare complication is hypertensive encephalopathy in the form of seizures. Patients who have a negative initial seizure workup otherwise should have an MRI. These patients need tight blood pressure control and seizure prophylaxis.

\section{Keywords}

Hypertensive emergency, anti-glomerular membrane disease, seizure

\section{INTRODUCTION}

Anti-glomerular basement membrane (anti-GBM) disease is a form of vasculitis where auto-antibodies attack antigens of the glomerular basement membrane leading to rapidly progressive glomerulonephritis. ${ }^{1}$ The 
complications surrounding this disease often circulate around renal and pulmonary pathologies. There is some mention of neurological complications associated with anti-GBM disease in literature, but the actual pathologies and mechanisms remain unclear. Some of the possible theories include cerebral vasculitis, metabolic encephalopathy, or posterior reversible encephalopathy syndrome (PRES). ${ }^{2}$ The pathogenesis of neurological symptoms in a patient with anti-GBM disease such as hypertensive emergency in the form of hypertensive encephalopathy, is clinically significant in patients with severe renal disease. Hypertensive encephalopathy is a diagnosis of exclusion thus involves extensive workup to rule out all other etiologies of neurologic dysfunction and BP dysregulation. ${ }^{3}$ Acute or chronic kidney disease, including anti-glomerular basement membrane disease (anti-GBM), are among well-known causes of hypertension and can lead to hypertensive emergencies. ${ }^{4,5}$ Here, we present a patient newly diagnosed with anti-GBM disease complicated by end-stage renal disease (ESRD) who had two hospital admissions for hypertensive emergency with seizures. Our work up, investigations, and treatment suggest hypertensive encephalopathy as the cause of seizures in our patient.

\section{CASE PRESENTATION}

This is a 30-year-old Caucasian male with biopsy confirmed glomerular basement disease diagnosed 3 months prior to presentation, with past medical history of tobacco use disorder. He received treatment with hemodialysis (HD), glucocorticoids, and oral cyclophosphamide for anti-GBM disease treatment, as well as valsartan, amlodipine, and carvedilol for blood pressure control. He presented to the ED for nausea and non-bloody non-bilious vomiting with associated frontal headaches and photophobia. His BP was 184/124 and physical exam showed right lower quadrant tenderness, but otherwise was unremarkable and without any focal neurological deficits. His course was complicated by two witnessed grand mal seizures in the ED lasting 45 seconds and 25 seconds respectively which were controlled with lorazepam and levetiracetam. He was admitted to the intensive care unit (ICU) where his BP was controlled by the addition of clonidine and bumetanide to his outpatient regimen. Amlodipine was discontinued on admission due to concern that it may have caused immune thrombocytopenic purpura (ITP) as he was thrombocytopenic (Table 1). He was eventually stabilized and kept in the hospital for 3 days for further work-up. He was ultimately discharged on valsartan, carvedilol, clonidine, minoxidil, bumetanide, and continued HD.

Two months later, the patient was brought in by emergency medicine services (EMS) after an unwitnessed fall and his second presumed seizure. At this time, he was still on HD three times per week (prednisone and cyclophosphamide had been discontinued) and his BP regimen was adjusted in the outpatient setting to losartan, carvedilol, clonidine, and minoxidil. His family heard a loud thud from another room and found him on the floor minimally responsive but awake. On EMS arrival, the patient was complaining of a headache and was noted to be mentally altered. His BP at that time was 218/130 and on route to the ED, EMS witnessed a grand mal seizure of one-minute duration. In the ED, his BP remained elevated at 209/137 and he was mildly confused and agitated, presumed due to post-ictal state. He had a GCS of 15, 5/5 muscle strength in all extremities, intact sensation to light touch, and no focal neurological deficits. He received multiple doses of lorazepam in the ED to combat seizure activity and was intubated for airway protection. He was then admitted to the ICU for further seizure work-up and blood pressure control with a nitroglycerin drip.

\section{INVESTIGATIONS AND DIFFERENTIAL DIAGNOSIS}

Initial differential diagnosis for the patient's new onset seizures included hemorrhagic stroke, ischemic stroke, new onset epilepsy, metabolic encephalopathy, hypertensive encephalopathy, hepatic encephalopathy, hypertensive emergency, acute drug/alcohol intoxication, and PRES. The medical work up of both seizure admissions was combined in the following sections for simplicity.

Neurology

Computerized tomography $(\mathrm{CT})$ of the brain without contrast on both admissions were negative for acute intracranial pathology. The ventricles and sulci were normal and symmetric bilaterally and there was no evidence of hemorrhage, infarction, or mass ruling out stroke or tumor. There were no signs of cerebral edema 
ruling out PRES. VEEG monitoring was performed for several continuous days during both admissions and was inconclusive. There were no asymmetries, no seizures, no epileptiform abnormalities, and no periodic patterns. There was mixed frequency slowing but the neurology team suspected this to be from sedation as the patient remained intubated and on mechanical ventilation. Neurology ultimately found no clear etiology for his seizures ruling out epilepsy. MRI was not obtained during either of his admissions.

\section{Nephrology}

Blood ethanol and urine toxicology were unremarkable. He continued to receive HD on a three times per week schedule. Repeat basic metabolic panel continued to show impaired renal function (Table 1). Mild fluid overload (possibly incorrect estimated dry weight) due to his severe kidney disease likely contributed to his uncontrolled BP at home.

\section{Hematology}

During the second admission, when the patient first presented with seizures there was suspicion for thrombotic microangiopathy possibly due to severe hypertension, ITP, or thrombotic thrombocytopenic purpura (TTP). Platelet count was 58,000 and lactate dehydrogenase was 359 but trended down. He had a negative thrombotic work-up (Table 1) consisting of normal ADAMTS13 activity, negative direct antiglobulin test, normal serum C4, normal serum C3, parasite smear, and iron studies. Lab results were notable for a high reticulocyte count and low haptoglobin suggestive of hemolysis. The hepatitis panel was negative on multiple occasions. Prior to discharge his platelet level stabilized and he was asymptomatic where we ultimately came to a diagnosis of thrombotic microangiopathy secondary to HTN.

\section{Infectious Disease}

Infectious disease was consulted as he had leukocytosis with a white count of 13,000 and lactic acidosis but no source of infection. Repeat CBCs showed that leukocytosis had resolved on its own. Elevated lactic acid resolved and was likely elevated due to seizure activity as opposed to infectious process. Blood cultures showed no growth through admission. One urinalysis showed some bacteria but also squamous cells thus we could not diagnose him with a UTI definitively. Lumbar puncture with CSF was considered but not done as there were no neurological deficits and the patient improved once BP was controlled. Ultimately, we did not suspect an infectious diagnosis to be contributing to this patient's case.

\section{Cardiology}

EKGs were obtained; one of them showed occasional PVCs and at one point he had a slightly prolonged QT at $503 \mathrm{~ms}$ but otherwise unremarkable. Troponin was mildly elevated but eventually down trended with elevation likely due to impaired clearance of troponin due to ESRD.

\section{TREATMENT}

Since an underlying cause of his seizures was not identified after thorough work-up, treatment focused on controlling the patient's BP with medications and optimizing his volume status with dialysis continuing ESRD treatments. There was concern that he may have been missing BP medication doses at home resulting in large increases in BP and hence, was advised to continue losartan, carvedilol, clonidine, and minoxidil with the clonidine being in the form of a catapres patch. Given the unknown etiology of seizures, although most likely from hypertensive emergency and subsequent encephalopathy, the patient was discharged home on levetiracetam to help prevent future seizures.

\section{DISCUSSION}

There are many etiologies that have been known to cause seizures. These include epilepsy, hemorrhagic stroke, cerebral hypoxia, metabolic derangement, thyroid disease, head trauma, CNS infection, vascular malformations, intoxication, poisoning and some of the rarer causes include brain tumors, hypertensive encephalopathy or PRES. ${ }^{6}$ 
Our patient had an extensive seizure work up during his hospital admissions. One of the most important tests to perform is VEEG as this provides insight as to the type of seizure a patient may be having and if there is underlying epilepsy. ${ }^{7,8}$ Continuous VEEG was performed on our patient during both admissions for seizures. Obtaining continuous VEEG monitoring or at least four separate VEEGs increases the chance of picking up underlying epileptic pathology by about $80-90 \% .{ }^{9,10}$ Continuous VEEG in our patient never identified any wave-forms suggestive of epilepsy. Neuroimaging is another crucial component of the seizure work up. Typically brain magnetic resonance imaging (MRI) is preferred over CT scan as MRI has higher sensitivity for epileptogenic lesions as well as having superior soft tissue contrast for identifying any other possible underlying pathology, but CT scans are often sufficient enough to make an accurate diagnosis. ${ }^{7,11,12}$ Our patient had CT scans of the brain on both presentations but showed no abnormality suggesting the cause of his seizures. In this patient CT scans of the brain were preferred over MRI originally given the patient acuity as CT scans can be obtained quicker than an MRI and are often sufficient in identifying any potential causes of seizures. ${ }^{7,8,13}$ Typically seizure work up can be continued with outpatient MRI; however, in our case it was deferred as CT brain and VEEG were normal and an MRI was not going to change our treatment plan especially since the patient did not have any additional seizures once his blood pressure was controlled.

Additional work up of seizures includes blood work to evaluate metabolic and thyroid issues and ECG and lumbar puncture to rule out hypoxic cerebral injury from syncope and infectious processes respectively. ${ }^{7,14,15}$ Although there were some abnormalities in his lab results there was nothing evident as the cause of his seizures (Table 1). ECGs were done on our patient and were insignificant. Moreover, since there were no infectious signs or symptoms and blood cultures never showed any growth of organisms, a lumbar puncture was not indicated.

Given the extensive negative neurologic, hematologic, and infectious workup, seizures in our patient likely occurred due to hypertensive encephalopathy from increased intracranial pressure. The cerebral perfusion pressure (CPP) of the brain has a narrow and sensitive window that is easily affected by changes in systemic BP. Normally when there is an increase in systemic BP the cerebral vasculature vasoconstricts to maintain $\mathrm{CPP}$ in physiologic range. In sudden increases of systemic BP or uncontrolled hypertension CPP is not maintained and can lead to cerebral edema resulting in neurologic dysfunction manifesting as seizures. ${ }^{16,17}$ After our patient's blood pressure was stabilized during admission, he did not have any additional seizures suggesting that the cause of his seizures was severely elevated blood pressures.

Acute or chronic kidney disease have been shown to cause hypertensive emergency and encephalopathy. ${ }^{4,5} 80$ $85 \%$ of patients with chronic kidney disease (CKD) have hypertension. ${ }^{18}$ Hypervolemia is due to decreased glomerular filtration rate (GFR), which results in sodium retention and extracellular fluid expansion. ${ }^{19}$ Furthermore, an overactive renin-angiotensin system due to renal ischemia can lead to increased angiotensin II and renin causing increased systemic BP, while uremia and decreased renal clearance can cause a neural reflex that increase levels of the vasoconstrictor endothelin. ${ }^{20}$ Some of the implicated renal diseases that can follow this physiology include acute glomerulonephritis, renal vascular disease, renal infarction, and renal failure. Anti-GBM disease is a rapidly progressive glomerulonephritis falling under this category.

Regarding treatment, initial management of this patient was aimed at controlling the seizure. Patients experiencing seizures without a history of epilepsy are typically treated with a single antiepileptic medication. ${ }^{21}$ Our patient received lorazepam to terminate the seizure and levetiracetam to prevent recurrence of seizures. Typically, anti-epileptic medications are not required for acute seizure management as seizures resolve on their own within a few minutes unless the patient is critically ill or has underlying metabolic derangements. ${ }^{22}$ Given that our patient was critically ill with renal disease and a dangerously elevated BP on presentation, levetiracetam was given to prevent further seizures, which has been shown to be more effective and have more long term benefits compared to other prophylactic seizure medications. ${ }^{16}$

Controlling BP in hypertensive emergency with hypertensive encephalopathy and ESRD varies by physician preference, although most of the treatments focus around IV antihypertensives for rapid control. ${ }^{23}$ Our patient's BP was effectively treated at the first seizure admission with clonidine and his PTA medications 
(losartan, carvedilol, and minoxidil). On the second seizure admission he required a nitroglycerin drip which is commonly used in hypertensive emergency and was effective at controlling his BP. ${ }^{23}$ We suspect the underlying cause of our patient's hypertensive episodes to be the frequently resistant nature of hypertension in patients with ESRD in combination with anti-GBM disease. Although he was given anti-epileptic medications as well, the patient did not have any additional seizures after his BP was controlled. In addition, with BP medication compliance as an outpatient, there have been no further reports of additional seizures since his last admission.

\section{CONCLUSION}

Our patient was newly diagnosed with anti-GBM disease and presented with the unique pathophysiology of hypertensive emergency and subsequent encephalopathy in the form of seizures. He was successfully stabilized and managed with strict blood pressure and seizure control. Our report brings attention to the complications that may arise with anti-GBM disease. Our patient has not had any recurrence of seizures implying that the cause of his seizures was most likely related to his hypertensive crises and that adequate $\mathrm{BP}$ control can prevent seizures in patients with anti-GBM disease.

\section{AUTHORSHIP}

MM: initiated this case report; performed patient chart review, performed literature review, and wrote first and final manuscript.

RK: performed literature review, assisted in writing and editing the manuscript.

SO: performed patient chart review and assisted in writing and editing this manuscript.

JM: performed literature review and assisted in writing this manuscript.

\section{CONFLICTS OF INTEREST}

The authors declare no conflicts of interest.

\section{CONSENT}

Written consent for publication of this case report and accompanying images was obtained from the patient.

\section{REFERENCES}

1. McAdoo SP, Pussey CD. Anti-Glomerular Basement Membrane Disease.Clinical Journal of the American Society of Nephrology . 2017;12(7):1162

2. Preul C, Gerth J, Lang S, et al. Cerebral Involvement in a Patient with Goodpasture's Disease due to Shortened Induction Therapy: A Case Report. Journal of Medical Case Reports . 2009;3:120.

3. Dinsdale HB. Hypertensive Encephalopathy. Stroke . 1982;13(5):717-719.

4. Whaley-Connell AT, Sowers JR, Stevens LA, et al. CKD in the United States: Kidney Early Evaluation Program (KEEP) and National and Nutrition Examination Survey (NHANES) 1999-2004. American Journal of Kidney Diseases . 2008;51(4):13-20.

5. Vaughan CJ, Delanty N. Hypertensive Emergencies. The Lancet . 2000;356(9227):411-417.

6. Peixoto AJ. Acute Severe Hypertension. The New England Journal of Medicine . 2019;381(19):18431852.

7. Krumholz A, Wiebe S, Gronseth G, Shinnar S, Levisohn P, Ting T, Hopp J, Shafer P, Morris H, Seiden L, Barkley G, French J. Practice Parameter: Evaluating an Apparent Unprovoked First Seizure in Adults (An Evidence Based Review): Report of the Quality of Standards Subcommittee of the American Academy of Neurology and the American Epilepsy Society. Neurology . 2007;69(21).

8. Fountain NB, Van Ness PC, Swain-Eng R, Tonn S, Bever CT, American Academy of Neurology, Epilepsy Measure Development Panel and the American Medical Association-Convened Physician Consortium for Performance Improvement Independent Measure Development Process. Report of the Quality Measurement and Reporting Subcommittee of the American Academy of Neurology. Neurology . 2011;76(1):94-9. 
9. King MA, Newton MR, Jackson GD, Fitt GJ, Mitchell LA, Silvapulle MJ, Berkovic SF. Epileptology of the First-Seizure Presentation: A CLinica, Electroencephalographic, and Magnetic Resonance Imaging Study of 300 Consecutive Patients. The Lancet . 1998;352(9133):1007-11.

10. Marsan CA, Zivin LS. Factors Related to the Occurrence of Typical Paroxysmal Abnormalities in the EEG Records of Epileptic Patients.Epilepsia . 1970;11(4):361-81.

11. Huff JS, Morris DL, Kothari RU, Gibbs MA. Emergency Department Management of Patients with Seizures: A Multicenter Study.Academic Emergency Medicine . 2001;8(6):622-628.

12. Duncan JS. Imaging and Epilepsy. Brain: A Journal of Neurology . 1997;120(2):339-77.

13. ACEP Clinical Policies Committee, Clinical Policies Subcommittee on Seizures. Clinical Policy: Critical Issues in the Evaluation and Management of Adult Patients Presenting to the Emergency Department with Seizures. Annals of Emergency Medicine . 2004;43(5):605-25.

14. Angus-Leppan H. First Seizures in Adults. BMJ . 2014;348(2470).

15. Kishk NA, Sharaf Y, Ebraheim AM, Baghdady Y, Alieldin N, Afify A, Eldamaty A. Interictal Cardiac Repolarization Abnormalities in people with Epilepsy. Epilepsy and Behavior . 2018;79:106-111.

16. Szaflarski JP, Sangha KS, Lindsell CJ, Shutter LA. Prospective, Randomized, Single-Blinded Comparative Trial of Intravenous Levetiracetam Versus Phenytoin for Seizure Prophylaxis.Neurocritical Care . 2009;(12):165-172.

17. Armstead WM. Cerebral Blood Flow Autoregulation and Dysautoregulation.Anesthesiology Clinics . 2016;34(3):465-477.

18. Stefanski A, Schmidt KG, Waldherr R, Ritz E. Early Increase in Blood Pressure and Diastolic Left Ventricular Malfunction in Patients with Glomerulonephritis. Kidney International . 1996;50(4):13216.

19. Stern A, Sachdeva S, Kapoor R, Singh J, Sachdeva S. High Blood Pressure in Dialysis Patients: Cause, Pathophysiology, Influence on Morbidity, Mortality and Management. Journal of Clinical and Diagnostic Research . 2014;8(6).

20. Maldonado M. Hypertension in End-Stage Renal Disease. Kidney International . 1998;54(68):67-72.

21. Glauser T, Ben-Menachem E, Bourgeois B, et al. Updated ILAE Evidence Review of Antiepileptic Drug Efficacy and Effectiveness as Initial Monotherapy for Epilipetic Seizures and Syndromes. Epilepsia . 2013;54(3):551-563.

22. Fields MC, Labovitz DL, French JA. Hospital-Onset Seizures: An Inpatient Study. JAMA Neurology . 2013;70(3):360-364.

23. Aronow WS. Treatment of Hypertensive Emergencies. Annals of Translational Medicine . 2017;5(1).

\section{Hosted file}

Table 1.docx available at https://authorea.com/users/332523/articles/458939-anti-glomerularbasement-membrane-disease-presenting-as-hypertensive-emergency-and-seizures 